ARTICLE

\title{
Observation of nanoscale opto-mechanical molecular damping as the origin of spectroscopic contrast in photo induced force microscopy
}

\author{
Mohammad A. Almajhadi (10 ${ }^{1,2}$, Syed Mohammad Ashab Uddin (iD ${ }^{1} \&$ H. Kumar Wickramasinghe (i) ${ }^{1 凶}$
}

Infrared photoinduced force microscopy (IR-PiFM) is a scanning probe spectroscopic technique that maps sample morphology and chemical properties on the nanometer (nm)-scale. Fabricated samples with $\mathrm{nm}$ periodicity such as self-assembly of block copolymer films can be chemically characterized by IR-PiFM with relative ease. Despite the success of IR-PiFM, the origin of spectroscopic contrast remains unclear, preventing the scientific community from conducting quantitative measurements. Here we experimentally investigate the contrast mechanism of IR-PiFM for recording vibrational resonances. We show that the measured spectroscopic information of a sample is directly related to the energy lost in the oscillating cantilever, which is a direct consequence of a molecule excited at its vibrational optical resonance-coined as opto-mechanical damping. The quality factor of the cantilever and the local sample polarizability can be mathematically correlated, enabling quantitative analysis. The basic theory for dissipative tip-sample interactions is introduced to model the observed opto-mechanical damping.

\footnotetext{
${ }^{1}$ Department of Electrical Engineering and Computer Sciences, University of California, Irvine, CA 92697, USA. ${ }^{2}$ Department of Electrical Engineering, University of Hail, Hail 2440, Saudi Arabia. ${ }^{凶}$ email: hkwick@uci.edu
} 
$\mathrm{T}$ he integration of atomic force microscopy (AFM) with focused lasers has enabled nano-chemical imaging and spectroscopy with spatial resolution well beyond the diffraction limit. One classic example is apertureless near-field scanning optical microscopy ${ }^{1-4}$. In this method, the enhanced optical field of the scanned AFM probe is perturbed by the local near field generated by the excited sample, and the scattered near field (amplitude and phase) is detected in the far field using an interferometer to record the image. Photothermal-induced resonance ${ }^{5,6}$ and peak force infrared (IR) ${ }^{7}$ are two examples for characterizing sample chemical properties based on AFM. In these techniques, the sample thermal expansion induced by optical absorption is detected using an AFM tip in contact. An alternative, noninvasive microscopy and spectroscopy technique that has emerged recently is photoinduced force microscopy $(\mathrm{PiFM})^{8}$ (Fig. 1). In this method, the tip-sample optical interaction is measured with the AFM operating in non-contact mode. The topography is recorded using the second mechanical eigenmode of the cantilever at $f_{2}$. A quantum cascade laser (QCL) is amplitude modulated at $f_{\mathrm{m}}$ (where $f_{\mathrm{m}}=f_{2}-f_{1}$ ) and focused on the tip end, and the opto-mechanical response is measured at the first mechanical eigenmode at $f_{1}$. Many applications of PiFM have emerged. Near-field electromagnetic field characterization ${ }^{9-14}$, nonlinear optical measurements such as Raman ${ }^{15}$ spectroscopy and stimulated Raman spectroscopy 16,17 , time-resolved pumpprobe microscopy ${ }^{18}$, organic solar cell studies ${ }^{19}$, optical phonon polariton imaging, and nanoscale chemical imaging in the mid$\mathrm{IR}^{20}$ are but a few examples.

While the dipole-dipole force model provides excellent agreement with the electromagnetic near-field measurements in the visible $^{14}$ and with mid-IR plasmonic resonance spectra ${ }^{21}$, extending this model to IR vibrational resonances causes discrepancies between experiment and theory ${ }^{20,22,23}$. In particular, the dipole-dipole force model predicts a dispersive spectral response, while the experimental results show a purely dissipative response. Three alternative proposals for explaining PiFM spectroscopic contrast in the IR have been proposed to address this discrepancy.
They are (1) detecting photothermal expansion using short-range repulsive forces acting on the AFM cantilever/tip 24,25 in contact, (2) detecting photoacoustic pressure waves generated at the sample surface resulting in long-range repulsive forces acting on the cantilever/tip ${ }^{25}$, and (3) detecting van der Waals (vdW)-mediated force modulation caused by sample thermal expansion ${ }^{26}$.

In this paper, we report on a series of experiments aimed at unraveling the origin of PiFM spectroscopic contrast in the IR. Our experimental findings support the hypothesis that the spectroscopic contrast in PiFM is mediated by opto-mechanical damping of the cantilever oscillation as the optical wavelength is scanned through optical resonance. Here the rate of dissipated mechanical power due to interactions induced by tip-sample dissipation processes can be divided into three components: $\gamma_{\text {ts }}$ due to adhesion, viscous damping, etc.; $\gamma_{\text {cant }}$ due to air or fluid damping; and $\gamma_{\text {opt }}$ due to opto-mechanical damping driven by dissipative near-field optical interaction. In our analysis, we assume that the opto-mechanical damping constant can be described by a velocity-dependent term in the Hamiltonian (as will be discussed later). The effective damping constant $\left(\gamma_{\text {eff }}\right)$ includes all damping effects $\left(\gamma_{\text {eff }}=\gamma_{\mathrm{ts}}+\gamma_{\mathrm{cant}}+\gamma_{\mathrm{opt}}\right)$, i.e., the total mechanical power dissipation increases upon optical absorption. We hypothesize that opto-mechanical damping force (change in energy with respect to distance) is caused by the excited sample molecules creating a dissipative force on the vibrating tip. We show that this contrast mechanism provides an excellent match with the experimental results. The theory can be extended to the single monolayer detection limit.

\section{Results}

Distinguishing between repulsive and attractive optical forces. Force gradients acting on an AFM tip shifts its dynamic stiffness $(k)$ and resonance frequency $f$. Figure 2 shows results from a 60nm-thick polystyrene (PS) film on gold (Au) substrate. We record the frequency shift of the cantilever at $f_{1}$ while the tip-sample gap is controlled at $f_{2}$. We plot the frequency shift at $f_{1}$ as we scan the a

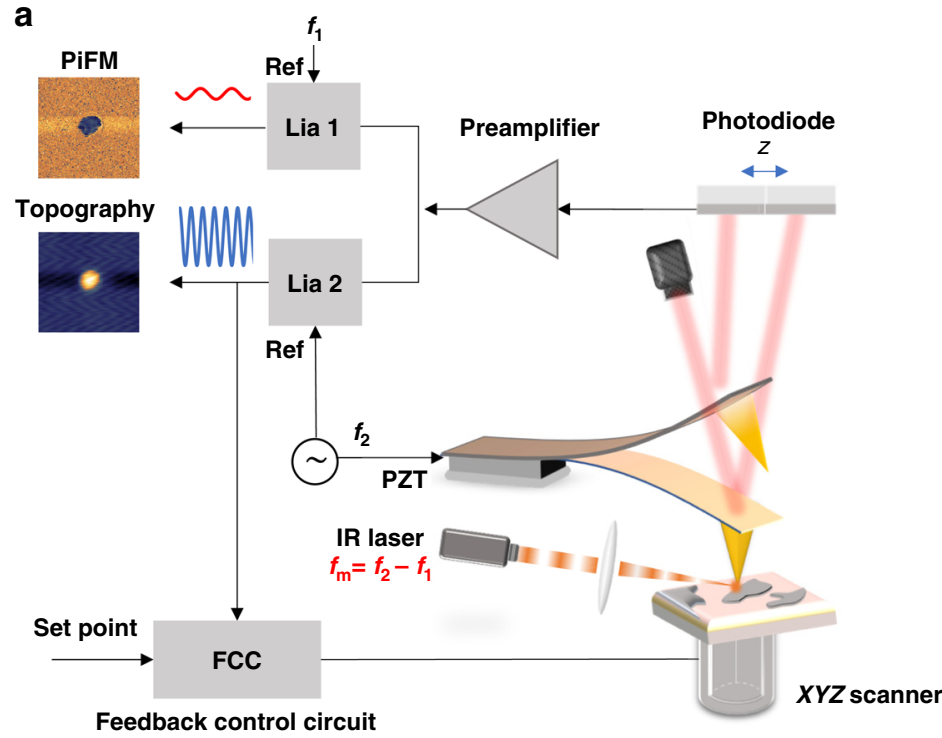

b

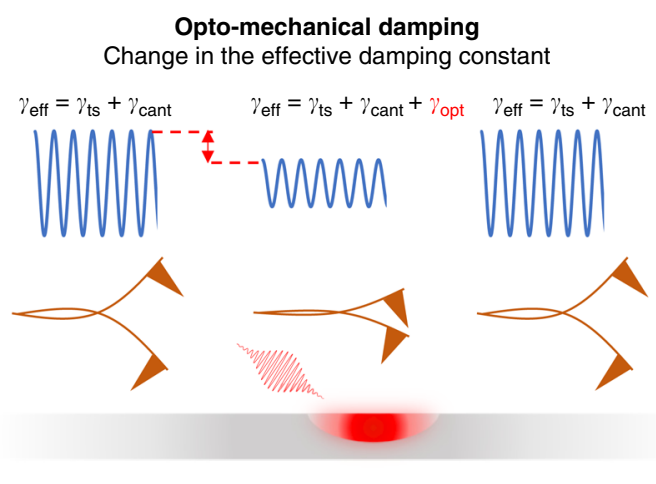

Fig. 1 Schematic of IR PiFM experiment and principle of opto-mechanical damping. a The cantilever is mechanically vibrated at its second mechanical eigenmode $f_{2}$, so that peak-peak oscillation is $6 \mathrm{~nm}$. Lock-in amplifier and feedback laser position sensitive detector (PSD) are used to stabilize the cantilever nanometers from sample surface. The IR source is electrically triggered at $f_{m}=f_{2}-f_{1}$, where $f_{1}$ is the first mechanical eigenmode of the cantilever. The incident infrared pulse is p-polarized (along the tip axis) and focused to 20- $\mu$ m-diameter spot. The topography and the PiFM signals are simultaneously recorded at $f_{2}$ and $f_{1}$, respectively. The image is generated via raster-scanning the sample under the tip. $\mathbf{b}$ illustrates the principle of optomechanical damping, where oscillation amplitude is damped due to IR vibrational resonance. 


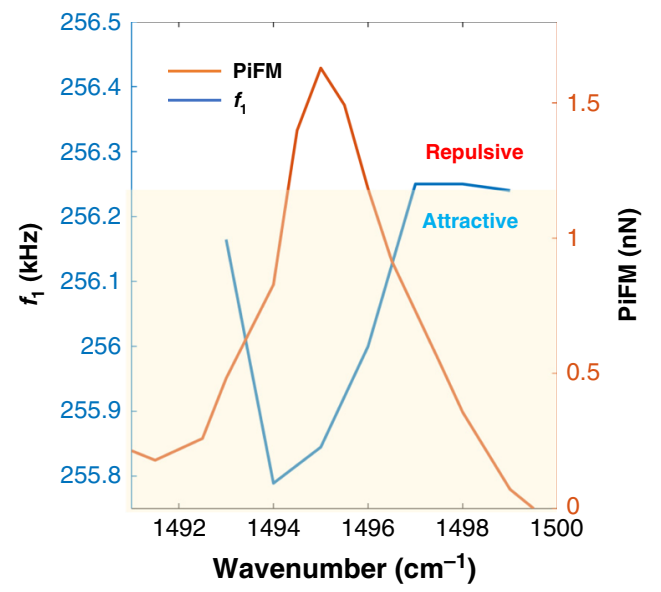

Fig. 2 Cantilever frequency shift across an absorption band. Shift of the cantilever resonance frequency at $f_{1}$ (blue line) across PS absorption band at $1495 \mathrm{~cm}^{-1}$ (orange line). The sample is $60-\mathrm{nm}$-thick PS film on Au

substrate. Input average power was $1 \mathrm{~mW}$ focused to 20 - $\mu \mathrm{m}$-diameter spot.

optical excitation wavelength through the PS resonance. When sample is excited on resonance, the frequency of the first eigenmode shows a maximum shift toward lower frequency values, relative to off resonance excitation, revealing the attractive nature of the optical force. Previous works ${ }^{27}$ have also come to the same conclusion, where the frequency shift was measured relative to the free oscillation amplitude (i.e., $3 \mu \mathrm{m}$ away from the sample surface); however, in those experiments, non-optical effects, such as van der Waal's (vdW) forces, were not subtracted. In our experiments, we automatically eliminate any vdW force gradient effects by measuring the frequency shift at $f_{1}$ as we scan through optical resonance while the tip is engaged. The observed frequency shifts were in the range of $500 \mathrm{~Hz}$. Therefore, short-range thermal expansion forces in contact (repulsive forces) are not relevant in PiFM.

It is well known that energy absorbed at the surface of a sample can generate acoustic pressure waves in the surrounding gasphotoacoustics ${ }^{28}$. For our system, the pressure waves will have a wavelength ranging from $248 \mu \mathrm{m}$ to $1.3 \mathrm{~mm}$ (corresponding to $f_{\mathrm{m}}=1.38 \mathrm{MHz}$ and $f_{1}=256 \mathrm{kHz}$ ). During one cycle of cantilever oscillation, the change in near-field photoacoustic force, i.e., acoustic force gradient, acting on the cantilever should be much smaller than the near-field optical force gradient acting on the tip. Photoacoustics generated by the $20 \mu \mathrm{m}$ IR spot on the sample could still exert a global repulsive force on the cantilever. We were indeed able to detect a global photoacoustic effect originating from the focused IR beam for relatively thick samples $(>200 \mathrm{~nm}$ ) (see Supplementary Fig. 1) but only when the tip is retracted a few $\mu \mathrm{m}$ from sample when the much larger optical forces become negligible. We also observed that the global photoacoustic signal disappears when the system is operated in a vacuum of 0.3 torr. Based on these considerations, we conclude that the near-field repulsive force due to gas photoacoustics will have minimal effect on the overall near-field PiFM signal in our measurements. Our experimental observations have refuted the proposals that gas photoacoustic forces or short-range thermal expansion forces play any significant role in PiFM contrast, at least in the regime that we have investigated-i.e., organic samples with thicknesses $<60 \mathrm{~nm}$. We will therefore no longer consider photothermal expansion or gas photoacoustics as potential contributors to hetrodyne PiFM contrast mechanism.

Piezo vibration experiments and PiFM sensitivity to thermal expansion. We showed that thermal expansion and photoacoustics (short- and long-range repulsive forces, respectively) do not play a significant role in our PiFM set-up. This leaves us with thermally modulated vdW forces $\left(F_{\mathrm{th}}^{\mathrm{vdW}}\right)$, i.e., thermal expansion modulates and amplifies the vdW force, which in turn acts on the AFM tip and consequently generates the PiFM signal. The modulated $F_{\mathrm{th}}^{\mathrm{vdW}}$ are long-range attractive forces. In this section, we mimic thermal expansion in our set-up by vibrating a mirrored lead zirconium titanate (PZT) crystalwhich in turn modulates the vdW force. Our PZT crystal was independently calibrated using a heterodyne laser interferometer (see Supplementary Fig. 2). Experiments were carried out to determine the smallest detectable thermal expansion in our PiFM.

Figure $3 \mathrm{~b}$ depicts our experimental set-up. Template-stripped gold (TSG) attached to PZT was vibrated at $f_{\mathrm{m}}=f_{2}-f_{1}$. The modulated vdW at $f_{\mathrm{m}}$ mixes with $f_{2}$ to generate a signal at $f_{1}$ due to nonlinear tip-sample interactions. We plotted the sensitivity $(S)$ defined as the ratio of the measured signal (pm) to the piezo displacement (pm) and compared it with the noise level of the PiFM to determine the minimum detectable thermal expansion. Results in Fig. 3a show a linear relationship between PZT displacement and the observed signal, with $S=A_{1} / d=1 \mathrm{pm} \mathrm{pm}^{-1}$, where $A_{1}$ is the peak-peak oscillation amplitude of the first mechanical mode and $d$ is the PZT displacement as shown in Fig. 3a (Fig. 3a will be used later to estimate the thermal expansion contribution to our PiFM signal in our monolayer experiments). Since the noise level measured at $f_{1}$ is about $32 \mathrm{pm}$ for $5 \mathrm{~ms}$ integration time (orange line in Fig. 3a, and see also Supplementary Fig. 4), thermal expansion below $32 \mathrm{pm}$ will not be detectable in our system. Thermal expansion for a $60-\mathrm{nm}$ PS film on silicon substrate (excited at $1452 \mathrm{~cm}^{-1}$ with $5 \mathrm{~mW}$ average power focused to 20 - $\mu \mathrm{m}$-diameter spot) has been previously calculated to be about $30 \mathrm{pm}$, which is already below our noise level ${ }^{26}$. That study shows that thermal signals generated by monolayer samples with typical thermal expansion of a few pm would be barely detectable. In the following section, the response of a 4-methylbenzenethiol (4-MBT) monolayer on TSG was measured with a signal-to-noise $(\mathrm{S} / \mathrm{N})$ ratio of 100 , further confirming that our PiFM contrast cannot be thermal or $F_{\mathrm{th}}^{\mathrm{vdW}}$ in origin.

Monolayer PiFM experiments. To demonstrate PiFM sensitivity to optical forces generated by molecular vibrational resonances of monolayer samples, 4-MBT self-assembled monolayer solution was prepared and TSG sample was immersed and left overnight in solution. The TSG is expected to be completely covered by a 4MBT monolayer. Gold islands are generated by sonicating the TSG in ethanol until gold starts lifting off. Figure $4 \mathrm{c}$ shows the topography of the sample. The thickness of the monolayer is $<5$ $\AA^{29}$. The sample was excited with p-polarized light using QCL. Measured average power was $0.5 \mathrm{~mW}$. The diameter of the focal spot is $20 \mu \mathrm{m}$, with incident angle of $30^{\circ}$ measured from sample surface. The average tip-sample distance was controlled at $f_{2}$ with dithering amplitude of $6 \mathrm{~nm}$ peak to peak. Set point was adjusted such that average tip-sample distance is approximately $9 \mathrm{~nm}$ (refer to Fig. 6b). Thus, minimum average tip-sample distance will be around $6 \mathrm{~nm}$. QCL repetition rate was tuned such that the lower sideband $f_{2}-f_{\mathrm{m}}$ coincided with $f_{1}$. The PiFM signal is enhanced by the quality factor $\left(Q_{1}\right)$ at $f_{1}$, which is about 435 (see Supplementary Fig. 4). In addition to the mechanical enhancement, the silicon cantilever/tip was coated with 60-nm-thick gold to locally enhance the electromagnetic field.

Figure $4 \mathrm{~b}$ shows the absorption spectrum of $4-\mathrm{MBT}$, which is centered at $1495 \mathrm{~cm}^{-1}$, with full width at half maximum of about $4 \mathrm{~cm}^{-1}$. This sharp absorption band is a typical signature of benzene ring mode. Figure $4 \mathrm{c}$, d, respectively, show simultaneously recorded topography and PiFM images; Fig. 4e is PiFM image when $4-\mathrm{MBT}$ is excited off resonance; it shows that the 
a

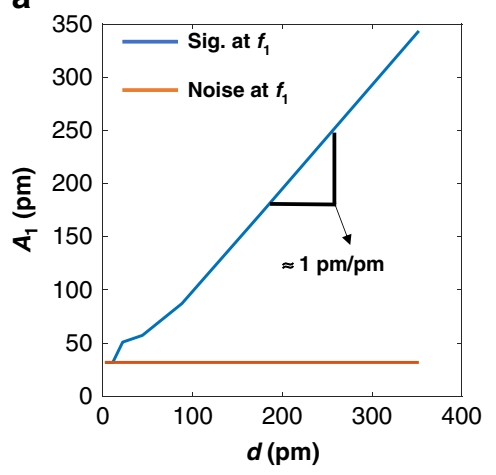

b

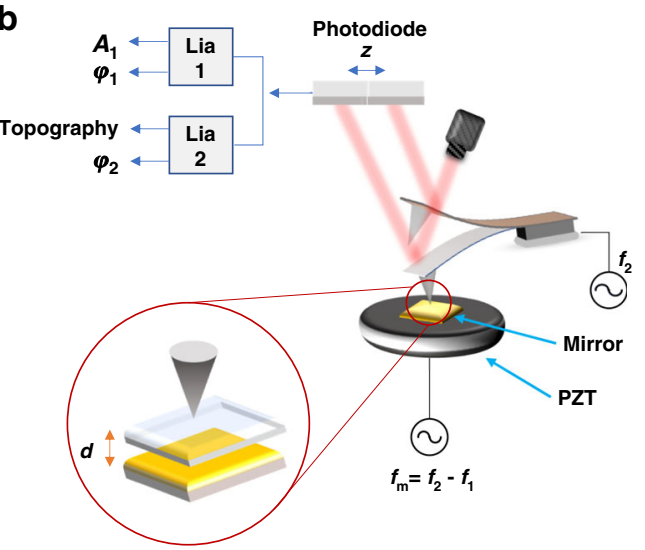

Fig. 3 Non-contact AFM sensitivity to thermal expansion. a The peak-peak oscillation amplitude (blue solid line) measured at $f_{1}$ as a function of the PZT displacement amplitude $(d)$. The noise level of the system measured at $f_{1}$ corresponds to oscillation of about 32 pm peak-peak as indicated by the orange line. Tip-sample gap is controlled at $f_{2}$ and the PZT is driven at $f_{m}=f_{2}-f_{1}$. b depicts the experimental set-up.

a

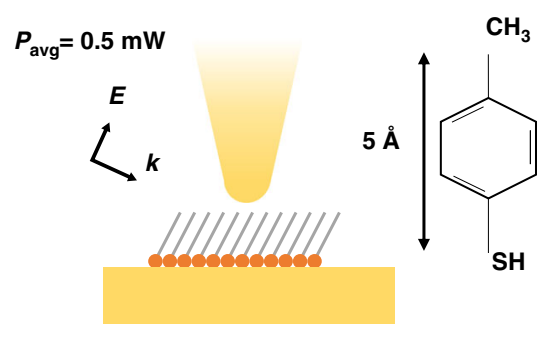

C

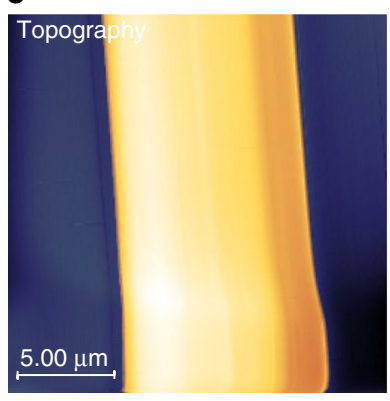

b

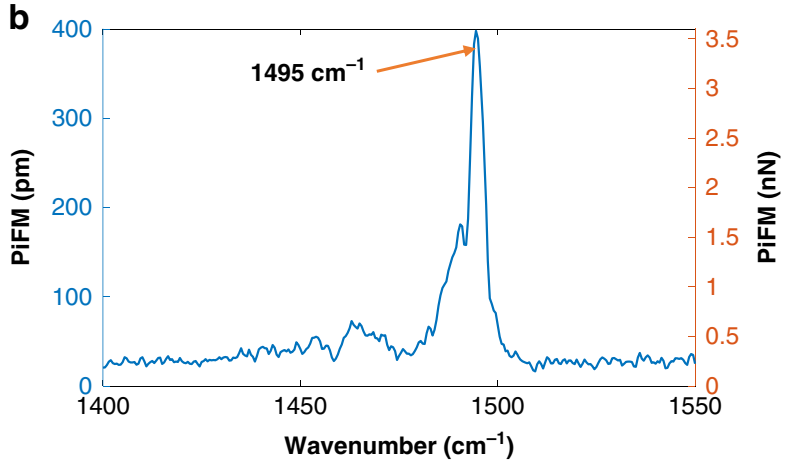

d

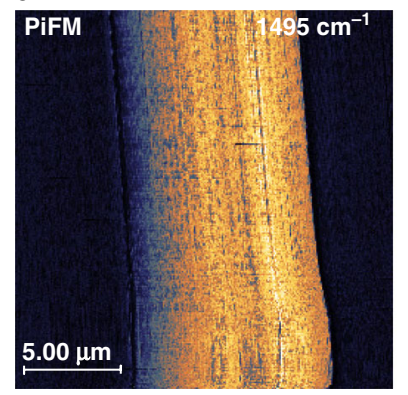

e

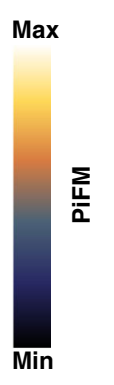

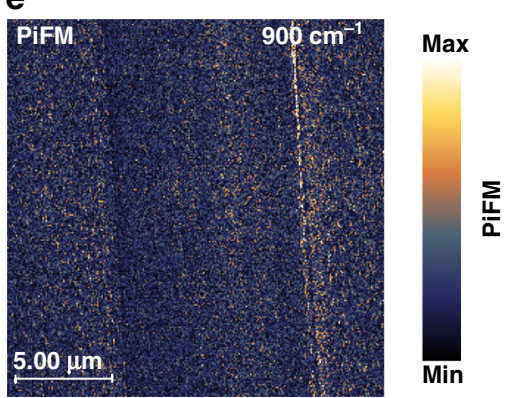

Fig. 4 Monolayer response. $\mathbf{a}$ is 4-MBT adsorbed on template-stripped gold. Excitation average power is $0.5 \mathrm{~mW}$ focused to 20 - $\mu \mathrm{m}$-diameter spot. $\mathbf{b}$ is point spectrum of $4-\mathrm{MBT}$ showing resonance at $1495 \mathrm{~cm}^{-1}$. c-e are topography of gold island, PiFM image at $1495 \mathrm{~cm}^{-1}$, and PiFM image off resonance, respectively.

signal observed in Fig. $4 \mathrm{~b}$ is not due to any possible crosstalk between topography and PiFM. Under similar experimental conditions, thermal expansion of a monolayer has been calculated numerically to be $<3 \mathrm{pm}$ with a temperature increase of $<6$ degrees $^{30,31}$. Also, we have shown in the previous section that minimum detectable thermal expansion is about $32 \mathrm{pm}$-limited by our system noise level. Because the maximum measured signal for 4-MBT corresponds to oscillation amplitude of $392 \mathrm{pm}$ peak-peak, and since $S=1 \mathrm{pm} \mathrm{pm}^{-1}$, the mono-molecular layer must expand $392 \mathrm{pm}$ to generate our signal-almost $100 \%$ of its initial thickness! Such an expansion would imply heating the molecular layer by several 100 s of degrees. We conclude that the observed PiFM signal clearly could not originate from thermal expansion. The findings also support the fact that any $F_{t h}^{\mathrm{vdW}}$ effect on the PiFM signal is negligible. In what follows, experiments were performed to study the effect of vibrational resonances on the cantilever dynamics and to unravel the actual contrast mechanism in IR-PiFM.

Opto-mechanical damping. The sidebands in PiFM can originate from either amplitude modulation (AM) or frequency modulation (FM) of the second resonance $f_{2}$ of the cantilever. In one analysis, it was considered to originate from a frequency modulation of $f_{2}$ resonance ${ }^{32}$; a change in tip-sample interaction force (force gradient) leads to change in the effective spring constant, which in turn shifts $f_{2}$ at the chopping frequency $f_{\mathrm{m}}$. Amplitude modulation of $f_{2}$ is another way to generate sidebands. The excited molecule interacting with the tip exerts a damping force in the tip leading to a change in the cantilever effective damping constant $\left(\gamma_{\mathrm{eff}}\right)$, which in turn amplitude modulates $f_{2}$ at $f_{\mathrm{m}}$. 
We conducted a series of experiments to determine whether the PiFM signal (mixed signal) detected at $f_{1}$ is due to AM or FM modulation of cantilever second eigenmode resonance. In our experiments, first the cantilever was mechanically excited (by the dithering PZT) at a frequency slightly higher than $f_{2}\left(f_{2 \mathrm{R}}\right)$ and then excited at a frequency slightly lower than $f_{2}\left(f_{2 \mathrm{~L}}\right)$. Note that $f_{2}$ is used as feedback signal (to control the average tip-sample distance). The sample was $60 \mathrm{~nm}$ poly(methyl methacrylate) (PMMA) on glass. The laser was tuned to a PMMA resonance and modulated at $f_{\mathrm{m}}=f_{2}-f_{1}$. For attractive conservative optical interactions, $A_{2}$ is expected to decrease when cantilever is mechanically excited at $f_{2 \mathrm{R}}$ and increase when excited at $f_{2 \mathrm{~L}}$; this is because the attractive forces shift the frequency to a lower value. Therefore, because the two behaviors are opposite to each other, the expected relative phase $\left(\varphi_{\text {rel }}=\left|\varphi_{1}^{f_{2 R}}-\varphi_{1}^{f_{2 L}}\right|\right)$ measured for PiFM signal is expected to be about $180^{\circ}$ out of phase. For dissipative optical interactions, the amplitude $A_{2}$ decreases for $f_{2 \mathrm{R}}$ and $f_{2 \mathrm{~L}}$, hence $\varphi_{\text {rel }}$ is about $0^{\circ}$. Thus the value of $\varphi_{\text {rel }}$ can be used to understand the nature of the interaction. When we measured $\varphi_{\text {rel }}$ of the PiFM signal at $f_{1}$ using a lock-in amplifier, we discovered that $\varphi_{\text {rel }}$ was nearly $0^{\circ}$ (see Supplementary Fig. 5) indicating that our PiFM signal contrast was originating from AM rather than FM modulation of the cantilever second eigenmode.

In order to confirm our finding by direct measurement, we perform the following experiment. The cantilever was mechanically excited at $f_{2 \mathrm{R}}$. The tip was approached and engaged with the sample. The feedback loop was opened, and the laser wavelength was rapidly swept across PMMA absorption band centered at $1733 \mathrm{~cm}^{-1}$; the oscillation amplitude $\left(A_{2 \mathrm{R}}\right)$ at $f_{2 \mathrm{R}}$ was recorded and compared with the point spectrum taken earlier for the same sample but with the control loop closed. The acquisition time needed to be fast enough to minimize thermal drift during data acquisition. In addition, laser modulation frequency $f_{\mathrm{m}}$ was set at $1 \mathrm{MHz}$ so that it did not excite any cantilever eigenmodes (see Supplementary Fig. 6) -i.e., in these studies, we can consider the laser to be behaving essentially as a continuous wave source of energy. Our experiments revealed that the mechanical oscillation amplitude of the cantilever $A_{2 \mathrm{R}}$ was damped as the laser was scanned through the PMMA resonance! (Fig. 5d). The experiment was repeated again with excitation frequency at $f_{2 \mathrm{~L}}$ with exactly the same result (Fig. 5f). Figure 5a. b show the expected phase and amplitude response of two harmonic oscillators with different quality factors. If photoinduced force was dissipative, the predicted amplitude behavior for $f_{2 \mathrm{R}}$ and $f_{2 \mathrm{~L}}$ is shown as a transition from $a$ to $a$ and from $b$ to $b$. As mentioned, $A_{2}$ decreases in both cases as shown in Fig. 5b, d, f, in contrast to what is expected from a conservative force. The change in $Q_{2}$ is evident and it tracks the change in the point spectrum.

Another piece of evidence that demonstrates the dissipative nature of photoinduced force is shown in the phase measurements of Fig. $5 c$, e. The phase of $f_{2}\left(\varphi_{2}\right)$ was recorded while the optical wavenumber was rapidly swept through resonance. Here the experiment is done with the feedback loop closed. According to Fig. $5 \mathrm{a}$, we should expect to observe a decrease in the phase for $f_{2 \mathrm{R}}$ and an increase at $f_{2 \mathrm{~L}}$ as verified in Fig. $5 \mathrm{c}$, e. The change in $\varphi_{2 \mathrm{R}}$ is $3^{\circ}$ at $f_{2 \mathrm{R}}$ and the change in $\varphi_{2 \mathrm{~L}}$ is $1^{\circ}$ at $f_{2 \mathrm{~L}}$. We note that the phase measurements and point spectrum were simultaneously recorded for the $60 \mathrm{~nm}$ PMMA film on glass.

As a further confirmation, we perform a direct measurement of the quality factor of the first mechanical mode $\left(Q_{1}\right)$. Here we have controlled the tip-sample gap using the second mechanical mode. The first mechanical mode is free (i.e., the amplitude and phase are not restricted by the feedback loop). Once we engaged with the sample, we excited the first eigenmode using dithering piezo to measure its quality factor with laser ON and OFF (i.e., both eigenmodes are excited mechanically using the dithering PZT). Similar to the previous experiment, we set $f_{\mathrm{m}}=1 \mathrm{MHz}$. To measure $Q_{1}$ at different average tip-sample distances, the frequency of the applied signal to the dithering PZT is tuned across $f_{1}$ and the oscillation setpoint of the feedback loop is lowered. For each setpoint, we measure $Q_{1}$ when we excite the sample on resonance (laser ON) and when the laser is OFF. $Q_{1}$ decreases at a faster rate when sample is excited ON resonance versus when laser is OFF (see Supplementary Fig. 9).

Based on all our experiments, we conclude that a change in $\gamma_{\text {eff }}$ of the cantilever rather than a change in its effective spring constant is the dominant contrast mechanism in PiFM. The intensity modulated excitation source modulates $\gamma_{\text {eff }}$ at $f_{\mathrm{m}}$, which in turn generates the mixing signal measured at $f_{1}=f_{2}-f_{\mathrm{m}}$.

Consider the tip-sample ensemble as spring-dashpot-mass system; the oscillation of the cantilever normal to sample surface ( $\mathbf{z}$ direction) can be approximately described by a non-linear, second-order differential equation

$$
m \frac{\mathrm{d}^{2} \mathbf{z}}{\mathrm{dt}^{2}}+m \gamma_{\text {eff }} \frac{\mathrm{d} \mathbf{z}}{\mathrm{dt}}+k \mathbf{z}=\mathbf{F}_{\mathbf{d}}+\mathbf{F}_{\mathrm{int}}+\mathbf{F}_{\mathbf{o p t}},
$$

where $m, k$, and $\gamma_{\text {eff }}$ are the effective mass, stiffness, and damping constant, respectively. $\mathbf{F}_{\text {int }}, \mathbf{F}_{\mathbf{o p t}}$, and $\mathbf{F}_{\mathbf{d}}$ are the nonlinear vdW tip-sample interactions, near-field tip-sample optical interactions, and deriving force, respectively. Velocity-dependent dissipative tip-sample interactions such as viscoelasticity and adhesion hysteresis are significant when imaging soft samples, such as polymers. Therefore, we propose that the optical energy absorbed by the sample could induce a measurable change in one or both of these dissipative channels leading to the observed optomechanical damping. Because viscoelasticity and adhesion hysteresis are velocity dependent ${ }^{33}$, we assume that the optomechanical damping is velocity dependent; thus the effective damping constant is the sum of the opto-mechanical damping constant $\gamma_{\mathrm{opt}}$ and the non-optical damping constant $\gamma_{\mathrm{cant}}$ and $\gamma_{\mathrm{ts}}$ :

$$
\gamma_{\text {eff }}=\gamma_{\mathrm{ts}}+\gamma_{\text {cant }}+\gamma_{\text {opt }} .
$$

In our experiments, $f_{2} \sim 1.65 \mathrm{MHz}$ and $Q_{2} \sim 551$. Then the damping coefficient $m_{2} \gamma_{\text {eff }}=k_{2}\left(\omega_{0} Q_{2}\right)^{-1}$ (where $m_{2}$ is the effective mass at $f_{2}$ ) is approximately $65 \mathrm{nN} \mathrm{s} \mathrm{m}^{-1}$. From Fig. 5e, $\mathrm{f}$, we see that the cantilever oscillation is reduced by approximately $35 \%$ when the tip is stabilized $9 \mathrm{~nm}$ from the sample surface and the optical wavelength is tuned to the molecular resonance. We conclude that $\gamma_{\mathrm{opt}}$ is $0.35 \times 65$ or $22 \mathrm{nN} \mathrm{s} \mathrm{m}^{-1}$ at molecular resonance. $\gamma_{\text {opt }}$ reaches its maximum value when the molecule is driven at its optical resonance (i.e., at maximum optical polarization of the molecule).

Theoretical modeling. In PiFM, we observe additional damping of the vibrating cantilever tip when the sample molecule is irradiated with photon energy corresponding to one of its molecular vibrational modes. We can model the increase due to optomechanical damping (Fig. 5) as follows ${ }^{34}$. The averaged mechanical power delivered to the cantilever by the dithering piezo at $\omega_{\mathrm{d}}$ is given by

$$
<P_{\text {in }}^{\text {mech }}>=\frac{1}{2} A A_{\mathrm{d}} k \omega_{\mathrm{d}} \sin (\phi),
$$

where $A$ and $A_{\mathrm{d}}$ are oscillation amplitude of the cantilever and oscillation amplitude of the dithering piezo, respectively. $k$ and $\Phi$ are stiffness of the cantilever and the phase difference between driving signal and cantilever oscillation, respectively. The power loss $\left\langle P_{\text {diss }}^{\text {cant }}\right\rangle$ in the cantilever with quality factor $Q$ and resonance 

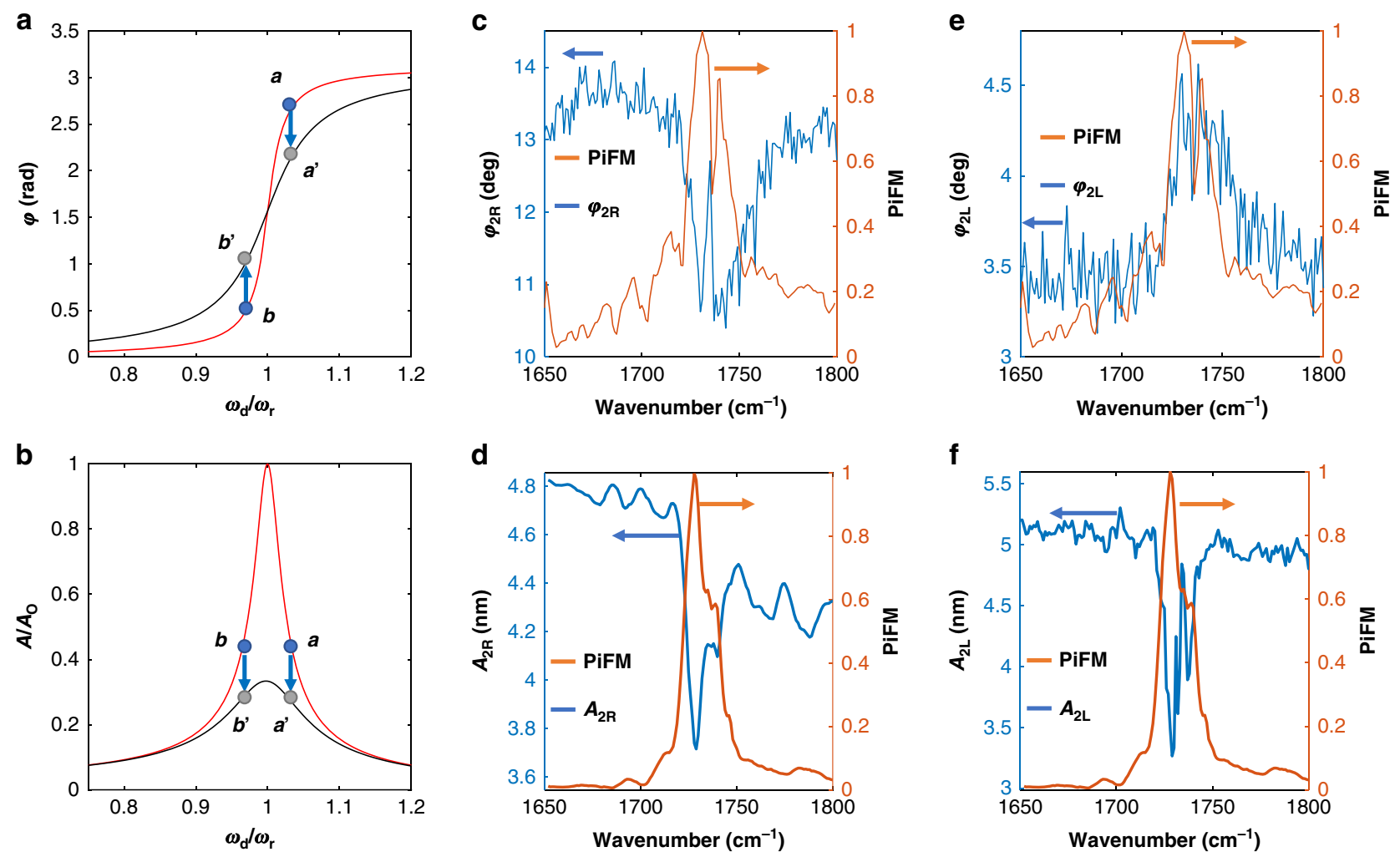

Fig. 5 Opto-mechanical damping. a, b are phase and amplitude of high $\mathrm{Q}$ (red) and low $\mathrm{Q}$ (black) harmonic oscillator. Change in the phase ( $\varphi_{2}$ ) and amplitude $\left(A_{2}\right)$ of the second mechanical mode (blue line) across PMMA absorption band (orange line) centered at $1733 \mathrm{~cm}^{-1} \mathrm{for}$ right excitation $(\mathbf{c}, \mathbf{d})$ and left excitation $(\mathbf{e}, \mathbf{f})$. Sample is 60-nm-thick PMMA on glass. The phase measurements were conducted using standard PiFM mixing mode, while amplitude measurements were conducted with the feedback loop open and the wavenumber scanned rapidly through resonance as explained in the text.

frequency $\omega_{\mathrm{d}}$ (primarily caused by air damping) is given by

$$
<P_{\text {diss }}^{\text {cant }}>=\frac{1}{2 Q} k A^{2} \omega_{d} .
$$

If $<P_{\text {diss }}^{\text {ts }}>$ and $<P_{\text {loss }}^{\text {opt }}>$ are the averaged power loss due to nonoptical tip-sample dissipative interactions and opto-mechanical damping of the cantilever, respectively, from power balance, we can write

$$
<P_{\text {diss }}^{\mathrm{ts}}>+<P_{\text {loss }}^{\mathrm{opt}}>=<P_{\text {in }}^{\mathrm{mech}}>-<P_{\text {diss }}^{\mathrm{cant}}>\text {. }
$$

Combining Eqs. (3)-(5) gives

$$
<P_{\text {diss }}^{\mathrm{ts}}>+<P_{\text {loss }}^{\mathrm{opt}}>=\frac{k A^{2} \omega_{\mathrm{d}}}{2 Q}\left(\frac{Q A_{\mathrm{d}} \sin (\emptyset)}{A}-1\right) .
$$

Solving for cantilever oscillation amplitude $A$ gives

$$
A=Q A_{\mathrm{d}} \sin (\emptyset)\left(\frac{\left.<P_{\mathrm{diss}}^{\mathrm{ts}}>+<P_{\text {loss }}^{\mathrm{opt}}\right\rangle}{\left.<P_{\mathrm{diss}}^{\mathrm{cant}}\right\rangle}+1\right)^{-1} .
$$

From Newton's equation of motion for a damped oscillating cantilever, we can relate rate of dissipated mechanical power $\left\langle P_{\text {diss }}^{\text {cant }}\right\rangle$ due to air damping to damping constant $\gamma_{\text {cant }}$ through $<P_{\text {diss }}^{\text {cant }}>=0.5 m \gamma_{\text {cant }} \omega_{\mathrm{d}}^{2} A^{2}$ (where $m$ is the effective mass of the cantilever); we can therefore write Eq. (5) in terms of $\gamma_{\text {opt }}$ and $\gamma_{\text {cant }}$.

$$
A=Q A_{\mathrm{d}} \sin (\emptyset)\left(\frac{\gamma_{\mathrm{ts}}+\gamma_{\mathrm{opt}}}{\gamma_{\text {cant }}}+1\right)^{-1} \text {. }
$$

Equations (7) and (8) show that the cantilever oscillation amplitude decreases when the opto-mechanical damping constant $\gamma_{\text {opt }}$ or the optically mediated tip-sample power dissipation increases-both will reach a maximum at optical resonance.
Modulating the incident light intensity at $f_{\mathrm{m}}\left(f_{\mathrm{m}}=f_{2}-f_{1}\right)$ produces maximum sideband oscillation amplitude and therefore maximum signal at $f_{1}$ when the sample is driven at one of its vibrational resonances. In addition, in order to get maximum sensitivity for detecting molecular resonance, we need to choose an AFM set-up with the lowest mechanical loss. $\mathrm{S} / \mathrm{N}$ ratio will be greatly improved by working even in a rough vacuum where air damping would be significantly minimized.

The $z$ component of the optical force $F_{\mathrm{tz}}$ acting on the tip with effective dipole moment $\mu_{\text {te }}$ can be written as ${ }^{33}$

$$
F_{\mathrm{tz}}=\mu_{\mathrm{te}} \frac{\mathrm{d} E_{\mathrm{tz}}}{\mathrm{d} z}
$$

or

$$
F_{\mathrm{tz}} \mathrm{d} z=\mu_{\mathrm{te}} \mathrm{d} E_{\mathrm{tz}}=\alpha_{\mathrm{te}} E_{\mathrm{tz}} \mathrm{d} E_{\mathrm{tz}} .
$$

Where $\alpha_{\text {te }}$ is the effective polarizability of the tip

$$
F_{\mathrm{tz}} \frac{\mathrm{d} z}{\mathrm{~d} t}=\alpha_{\mathrm{te}} E_{\mathrm{tz}} \frac{\mathrm{d} E_{\mathrm{tz}}}{\mathrm{d} t} .
$$

The time-averaged mechanical power $<P_{\text {loss }}^{\text {opt }}>$ dissipated due to optical forces acting on the tip is

$$
<P_{\text {loss }}^{\mathrm{opt}}>=<F_{\mathrm{tz}} \frac{\mathrm{d} z}{\mathrm{~d} t}>=0.5 \operatorname{Re}\left[\alpha_{\mathrm{te}}^{*} E_{\mathrm{tz}}^{*} \mathrm{i} \omega_{0} E_{\mathrm{tz}}\right]=0.5 \operatorname{Im}\left[\alpha_{\mathrm{te}}^{*} E_{\mathrm{tz}}^{*} \omega_{0} E_{\mathrm{tz}}\right],
$$

where $\omega_{0}$ is the optical frequency and $E_{\mathrm{tz}}$ the $z$-component of the electric field at tip where

$$
E_{\mathrm{tz}}=\left(1+\frac{\alpha_{\mathrm{s}}}{2 \pi\left(d+2 a_{\mathrm{t}}\right)^{3}}\right) E_{\mathrm{i}}
$$


and $\alpha_{\mathrm{te}}$ is given by ${ }^{34}$

$$
\alpha_{\mathrm{te}} \sim \alpha_{\mathrm{t}}+\frac{\alpha_{\mathrm{t}} \alpha_{\mathrm{t}} \beta_{\mathrm{s}}}{16 \pi\left(d+2 a_{\mathrm{t}}\right)^{3}} .
$$

$\alpha_{\mathrm{t}}$ and $\alpha_{\mathrm{s}}$ are the polarizabilities of tip and sample, respectively, $d$ is average tip-sample distance, $a_{\mathrm{t}}$ is tip radius, and $E_{\mathrm{i}}$ is the incident field.

To arrive at Eq. (14), we made the approximation $\left[\alpha_{\mathrm{t}}\left(1-\frac{\alpha_{\mathrm{s}} \beta_{\mathrm{s}}}{16 \pi\left(d+2 a_{\mathrm{t}}\right)^{3}}\right)^{-1}\right] \sim\left[\alpha_{\mathrm{t}}+\frac{\alpha_{\mathrm{t}} \alpha_{\mathrm{t}} \beta_{\mathrm{s}}}{16 \pi\left(d+2 a_{\mathrm{t}}\right)^{3}}\right]$.

Using Eqs. (10)-(14), $<P_{\text {loss }}^{\mathrm{opt}}>$ can be written as

$$
<P_{\text {loss }}^{\text {opt }}>\sim \frac{1}{2} \Im\left(a_{\mathrm{t}}^{*} E_{\mathrm{i}}^{2}\right)+\frac{1}{2} \Im\left(\frac{\omega_{\mathrm{o}} a_{\mathrm{t}}^{*}\left(a_{\mathrm{s}}^{*}+a_{\mathrm{s}}\right) E_{\mathrm{i}}^{2}}{2 \pi\left(d+2 a_{\mathrm{t}}\right)}\right)+\frac{1}{2} \Im\left(\frac{\omega_{a_{\mathrm{t}}}^{*} a_{\mathrm{s}}^{*} \beta_{\mathrm{s}}^{*} E_{\mathrm{i}}^{2}}{16 \pi\left(d+2 a_{\mathrm{t}}\right)^{3}}\right),
$$

where $\beta_{\mathrm{s}}=\left(\epsilon_{\mathrm{s}},-1\right) /\left(\epsilon_{\mathrm{s}},+1\right)$ is sample reflection coefficient, with $\epsilon_{\mathrm{s}}$ the complex dielectric function of the tip. Tip polarizability $\alpha_{\mathrm{t}}=\xi V_{\mathrm{t}}\left(\epsilon_{\mathrm{t}},-1\right) /\left(\epsilon_{\mathrm{t}},+2\right)$, with $V_{\mathrm{t}}$ the effective tip volume, $\xi$ is tip field enhancement factor when tip is far from surface, and $\epsilon_{\mathrm{t}}$ is the complex dielectric function of tip. We have a similar expression for $\alpha_{\mathrm{s}}$ with the parameters for sample replacing those of tip, except that $\xi=1$ in the latter case. Based on our numerical simulations for the gold-coated tip, $\xi \sim 10$. The last term in Eq. (15) is the most dominant term.

Equation 15 was evaluated with the appropriate Lorentzian dielectric functions for PMMA and dielectric constants for the tip ${ }^{35,36}$. Figure $6 \mathrm{~d}$ compares the experimental point spectrum (diamond symbol) with Eq. 15 (solid line) showing excellent agreement. For the PiFM approach curve, $f_{1}$ was mechanically tuned to get the maximum PiFM signal for each setpoint (as shown in Fig. 6c). The tracked peaks were normalized to the corresponding $A_{2}$. Figure $6 \mathrm{~b}$ shows the PiFM signal and the corresponding $A_{2}$ signal as a function of average tip-sample distance. We see that the PiFM signal is measurable up to $18 \mathrm{~nm}$ from sample surface. Figure 6e shows a fit to the experimental approach curve using Eq. (16). The data fits a $d^{-3}$ dependence as expected up to a tip-sample distance of $5 \mathrm{~nm}$. However, the PiFM signal shows a characteristic feature at molecular resonance; the signal increases monotonically as the tip approaches the sample but then decreases at the inflection point at $4 \mathrm{~nm}$.

The inflexion point can be incorporated into our model by adding an additional complex distance $-\mathrm{j} b$ to the distance dependence ${ }^{37,38}$.

$$
<P_{\text {loss }}^{\text {opt }}>\sim \frac{1}{2}\left[\Im\left(\omega_{\mathrm{o}} a_{\mathrm{t}}^{*}\right) E_{\mathrm{i}}^{2}+\Im\left(\frac{\omega_{\mathrm{o}} a_{\mathrm{t}}^{*}\left(a_{\mathrm{s}}^{*}+a_{\mathrm{s}}\right) E_{\mathrm{i}}^{2}}{2 \pi\left[\left(d+2 a_{\mathrm{t}}-\mathrm{j} b\right)\right]^{3}}\right)+\Im\left(\frac{\omega_{\mathrm{o}} a_{\mathrm{t}}^{*} a_{\mathrm{t}}^{*} \beta_{\mathrm{s}}^{*} E_{\mathrm{i}}^{2}}{16 \pi\left[\left(d+2 a_{\mathrm{t}}-\mathrm{j} b\right)\right]^{3}}\right)\right] .
$$

While the overall force is attractive, this modification generates an additional distance-dependent repulsive term varying as $-\left(d+2 a_{\mathrm{t}}\right)^{-4}$. Similar distance dependences have been predicted in models of the interaction energy of an atomic dipole oscillating close to a conducting sphere ${ }^{39}$. However, as we shall point out in the "Discussion" section, such a distance-dependent loss of signal at tip-sample spacings $<9 \mathrm{~nm}$ can be due to different loss mechanisms.

The modified Eq. (16) was evaluated with tip radius $a_{\mathrm{t}}=12$ $\mathrm{nm}$, sample radius $a_{\mathrm{s}}=5 \mathrm{~nm}$, and the fitting parameter representing loss strength $b=5.9 \mathrm{~nm}$. The tip and sample radii were extracted experimentally by imaging grains on gold surface (see Supplementary Fig. 7). This modification generated an a
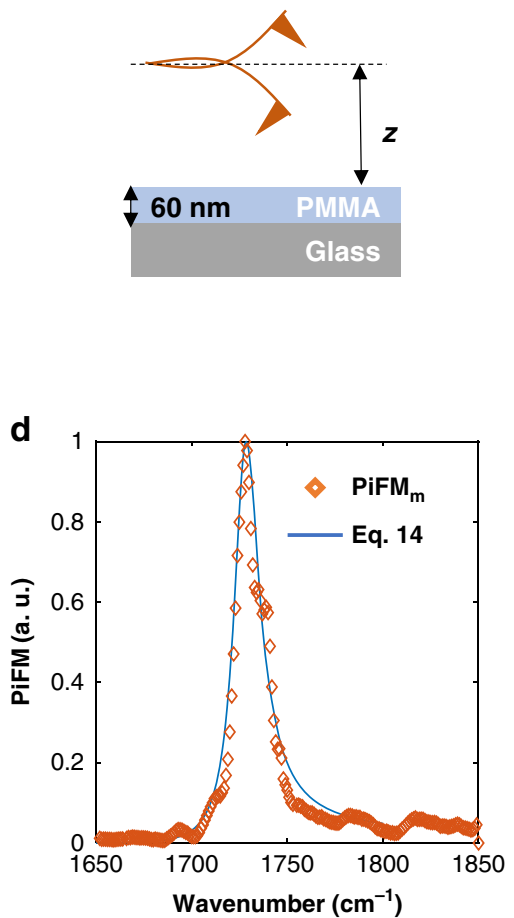

b
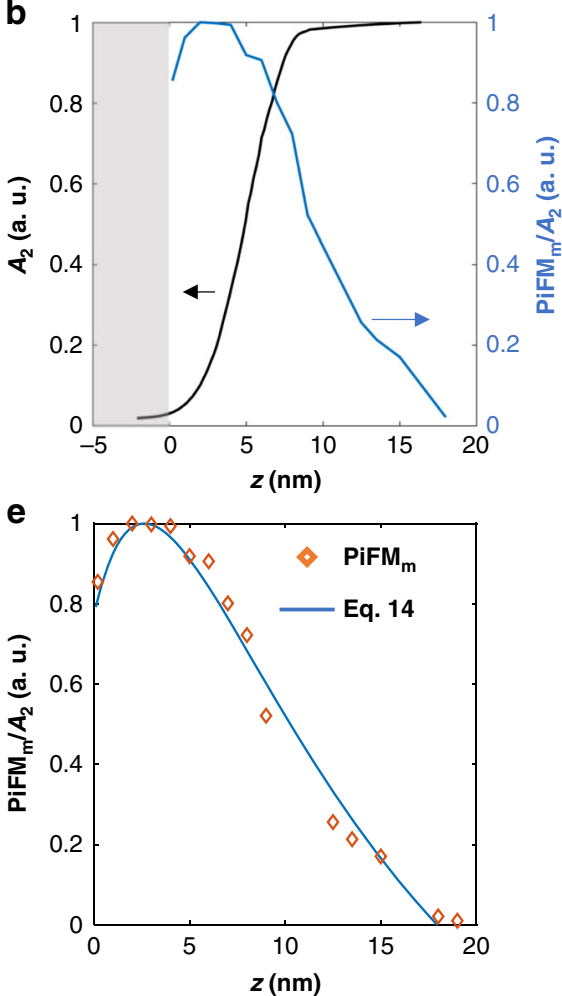

C

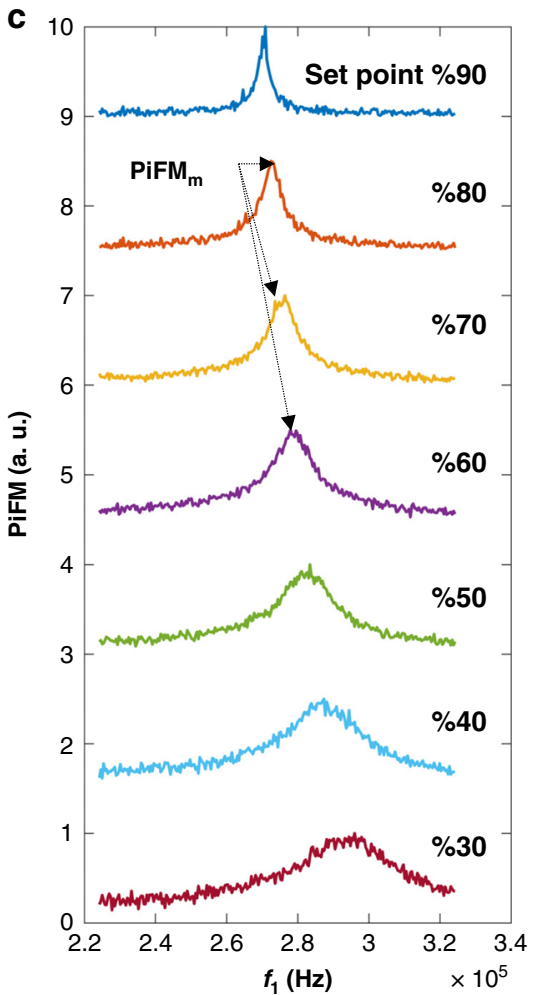

Fig. 6 Experimental and theoretical results of opto-mechanical damping. a Schematic of the tip and sample. b Amplitude $A_{2}$ measured at $f_{2}$ (black line) and maximum value of PiFM signal (PiFM $\mathrm{m}_{\mathrm{m}}$ ) measured at $f_{1}$ (blue line) as a function of average tip-sample distance. The shift in the peak value of $f_{1}$ as a function of average tip-sample distance is tracked in $\mathbf{c}$ and normalized by the correspondent amplitude measured at $f_{2}$. The set point for each curve in $\mathbf{c}$ is indicated as percentage of the amplitude of the cantilever oscillation at $f_{2}$. d, e are comparisons of the experiment (diamond) and Eq. (15) and Eq. 16 (solid line) for point spectrum and approach curve, respectively. 
excellent fit between our model and the measured PiFM approach curve (Fig. 6e solid line).

\section{Discussion}

Illuminating the cantilever with light could induce a temperature increase on the cantilever body. This increase in temperature can reduce the quality factor of the cantilever. A recent study showed that illuminating gold-coated cantilever near plasmonic resonance caused a measurable shift in the cantilever stiffness and a decrease in the quality factor ${ }^{40}$. Clearly this effect is not localized, and the temperature of the cantilever body has to increase for the quality factor to decrease. However, to extract nanoscale spectroscopic information of a sample, the opto-mechanical damping must originate and be localized within tip-sample volume of interaction, limited to the effective tip radius. The localization of the observed opto-mechanical damping is evident from Fig. $5 \mathrm{~d}$, $\mathrm{f}$, where the change in $A_{2}$ clearly follows the molecular resonance. Additionally, Fig. $6 \mathrm{~b}$ shows that the opto-mechanical damping is measurable up to $20 \mathrm{~nm}$ from sample surface at best. This vertical confinement (normal to sample surface) is another piece of evidence that the observed opto-mechanical damping must originate from localized interactions.

The proposed mechanism of PiFM contrast is modeled as optically mediated damping of the viscoelastic or adhesion interactions between tip and sample. For example, when the tip approaches close to the sample, the high electric fields in the optical nanocavity will tend to align the molecules along the axis of the tip. As the tip is retracted from the sample, the molecules will tend to relax back toward their equilibrium position (position without any applied field). These periodic molecular relaxations can occur over a time comparable to the tip oscillation period but with a different phase shift as compared to the tip oscillation phase. In another scenario, the vibrating molecule could enhance adhesion hysteresis causing an additional mechanical loss. Note that both viscoelastic and adhesion loss mechanisms are velocity dependent ${ }^{33}$. Since optomechanical damping is assumed to originate from one or both mechanisms, modeling opto-mechanical damping as velocitydependent dissipative process is justified.

Other possible mechanisms might contribute to the overall opto-mechanical damping. For example, Person et al. analyzed the effect of electron-hole (e-h) pair excitation on the life time of a vibrating point dipole near flat metal surface ${ }^{38,41}$. When a vibrating point dipole is located at a distance $d$ from semi-infinite metal, inelastic interaction can occur, and part of the interaction energy is radiated away from the system through photon emission and the other part excites e-h pair in the metal, which is a nonradiative loss channel. This lossy interaction quenches the dipole moment of the vibrating molecule and is responsible for the repulsive term in the interaction Hamiltonian. The dipole strength was shown to increase then decrease as a function of $d$, showing a hump located a few angstroms from metal surface ${ }^{37}$, similar to what we observe in Fig. 6b. Note that modeling excited molecule with finite-dipole moment instead of point-dipole moment, and replacing flat metal surface with sharp metallic tips, spatially extends near-field gradient of the electromagnetic field, and including these two effects alters the distance dependence of the damping effect and could shift the hump a couple of nanometers away from sample surface.

Another mechanism for opto-mechanical loss could be due to the fact that molecule in the optical cavity is driven with very high fields. This will result in the energy in the driven molecular mode being distributed to other modes within the cavity resulting in reduced response at the driven molecular mode. There is some evidence of splitting of optical resonance spectra when driven with high optical power. This effect will require detailed investigation in a future article.

\begin{abstract}
Methods
PiFM measurements. A commercial AFM (Molecular Vista Inc.) was used in noncontact mode for PiFM measurement. A pulsed QCL, from Block Engineering, was electrically triggered at $f_{\mathrm{m}}=f_{2}-f_{1}$ and focused to 20 - $\mu$ m-diameter spot; $f_{1}$ and $f_{2}$ are the first and second mechanical eigenmodes. QCL tuning range was from 800 to $1800 \mathrm{~cm}^{-1}$. The cantilever type used was PPP-NCHR-W $300 \mathrm{kHz}$ from Nanosensors, where $f_{1}=264 \mathrm{kHz}$ and $f_{2}=1.65 \mathrm{MHz}$. Quality factor $(Q)$ of the first and second mechanical modes were $Q_{1}=435$ and $Q_{2}=551$, respectively.
\end{abstract}

4-MBT self-assembled monolayer. A TSG was prepared and immersed for $24 \mathrm{~h}$ in $0.3 \mathrm{mM} 4$-MBT (Sigma Aldrich) in ethanol. This was followed by sonicating the TSG till some of the gold surface started to lift-off; an island of Au were formed.

PZT calibration. A heterodyne laser Interferometer was used to calibrate the vibrational expansion behavior of the PZT crystal (STEMINC). The arrangement consisted of a laser beam, beam splitters, Bragg cell, objective lenses, photodetector, high-gain low-noise amplifier, and a spectrum analyzer. HeNe laser source $(632 \mathrm{~nm})$ was used. The initial beam was split using a beam splitter. A portion was directed through a Bragg cell that shifted the optical frequency by $80 \mathrm{MHz}$. This beam acted as the reference beam of the interferometer. The other portion-the signal beam-was focused and reflected off the vibrating sample surface (the PZT crystal) generating a phase modulation proportional to the surface vibration amplitude.

\section{Data availability}

The data that support the findings of this study are available from the corresponding author upon reasonable request.

Received: 4 October 2019; Accepted: 14 September 2020; Published online: 10 November 2020

\section{References}

1. Wickramasinghe, H. K. \& Williams, C. C. Apertureless near field optical microscope. US patent 4,947,034 (1990).

2. Zenhausern, F., O’Boyle, M. P. \& Wickramasinghe, H. K. Apertureless nearfield optical microscope. Appl. Phys. Lett. 65, 1623-1625 (1994).

3. Knoll, B. \& Keilmann, F. Near-field probing of vibrational absorption for chemical microscopy. Nature 399, 134-137 (1999).

4. Zenhausern, F., Martin, Y. \& Wickramasinghe, H. K. Scanning interferometric apertureless microscopy: optical imaging at 10 angstrom resolution. Science 269, 1083-1085 (1995).

5. Dazzi, A., Glotin, F. \& Carminati, R. Theory of infrared nanospectroscopy by photothermal induced resonance. J. Appl. Phys. 107, 124519 (2010).

6. Dazzi, A., Prazeres, R., Glotin, F. \& Ortega, J. M. Local infrared microspectroscopy with subwavelength spatial resolution with an atomic force microscope tip used as a photothermal sensor. Opt. Lett. 30, 2388-2390 (2005).

7. Wang, L. et al. Nanoscale simultaneous chemical and mechanical imaging via peak force infrared microscopy. Sci. Adv. 3, e1700255 (2017).

8. Rajapaksa, I., Uenal, K. \& Wickramasinghe, H. K. Image force microscopy of molecular resonance: a microscope principle. Appl. Phys. Lett. 97, 073121 (2010).

9. Tumkur, T. et al. Wavelength-dependent optical force imaging of bimetallic Al-Au heterodimers. Nano Lett. 18, 2040-2046 (2018).

10. Jahng, J. et al. Visualizing surface plasmon polaritons by their gradient force. Opt. Lett. 40, 5058-5061 (2015).

11. Zeng, J. et al. Sharply focused azimuthally polarized beams with magnetic dominance: near-field characterization at nanoscale by photoinduced force microscopy. ACS Photonics 5, 390-397 (2018).

12. Tumkur, T. U. et al. Photoinduced force mapping of plasmonic nanostructures. Nano Lett. 16, $7942-7949$ (2016).

13. Rajaei, M., Almajhadi, M. A., Zeng, J. \& Wickramasinghe, H. K. Near-field nanoprobing using Si tip-Au nanoparticle photoinduced force microscopy with 120:1 signal-to-noise ratio, sub-6-nm resolution. Opt. Express 26, 26365-26376 (2018).

14. Huang, F., Ananth Tamma, V., Mardy, Z., Burdett, J. \& Kumar Wickramasinghe, H. Imaging nanoscale electromagnetic near-field distributions using optical forces. Sci. Rep. 5, 10610 (2015).

15. Rajapaksa, I. \& Wickramasinghe, H. K. Raman spectroscopy and microscopy based on mechanical force detection. Appl. Phys. Lett. 99, 161103 (2011). 
16. Tamma, V. A., Beecher, L. M., Shumaker-Parry, J. S. \& Wickramasinghe, H. K. Detecting stimulated Raman responses of molecules in plasmonic gap using photon induced forces. Opt. Express 26, 31439-31453 (2018).

17. Tamma, V. A., Huang, F., Nowak, D. \& Wickramasinghe, H. K. Stimulated Raman spectroscopy and nanoscopy of molecules using near field photon induced forces without resonant electronic enhancement gain. Appl. Phys. Lett. 108, 233107 (2016).

18. Jahng, J. et al. Ultrafast pump-probe force microscopy with nanoscale resolution. Appl. Phys. Lett. 106, 083113 (2015).

19. Gu, K. L. et al. Nanoscale domain imaging of all-polymer organic solar cells by photo-induced force microscopy. ACS Nano 12, 1473-1481 (2018).

20. Nowak, D. et al. Nanoscale chemical imaging by photoinduced force microscopy. Sci. Adv. 2, e1501571 (2016).

21. Huang, Y. et al. Spectroscopic nanoimaging of all-semiconductor plasmonic gratings using photoinduced force and scattering type nanoscopy. ACS Photonics 5, 4352-4359 (2018).

22. Ladani, F. T. \& Potma, E. O. Dyadic Green's function formalism for photoinduced forces in tip-sample nanojunctions. Phys. Rev. B 95, 205440 (2017).

23. Almajhadi, M. \& Wickramasinghe, H. K. Contrast and imaging performance in photo induced force microscopy. Opt. Express 25, 26923-26938 (2017)

24. Yang, H. U. \& Raschke, M. B. Resonant optical gradient force interaction for nano-imaging and -spectroscopy. New J. Phys. 18, 053042 (2016).

25. O’Callahan, B. T., Yan, J., Menges, F., Muller, E. A. \& Raschke, M. B. Photoinduced tip-sample forces for chemical nanoimaging and spectroscopy. Nano Lett. 18, 5499-5505 (2018).

26. Jahng, J., Potma, E. O. \& Lee, E. S. Tip-enhanced thermal expansion force for nanoscale chemical imaging and spectroscopy in photoinduced force microscopy. Anal. Chem. 90, 11054-11061 (2018).

27. Murdick, R. A. et al. Photoinduced force microscopy: a technique for hyperspectral nanochemical mapping. Jpn. J. Appl. Phys. 56, 08 LA04 (2017).

28. Tam, A. C. Applications of photoacoustic sensing techniques. Rev. Mod. Phys. 58, 381-431 (1986).

29. Streitwieser, A., Heathcock, C. H. \& Kosower, E. M. Introduction to Organic Chemistry (Prentice Hall, 1992).

30. Lu, F., Jin, M. \& Belkin, M. A. Tip-enhanced infrared nanospectroscopy via molecular expansion force detection. Nat. Photonics 8, 307-312 (2014).

31. Jin, M. \& Belkin, M. A. Infrared vibrational spectroscopy of functionalized atomic force microscope probes using resonantly enhanced infrared photoexpansion nanospectroscopy. Small Methods 3, 1900018 (2019).

32. Jahng, J., Kim, B., Lee, E. S. \& Potma, E. O. Quantitative analysis of sideband coupling in photoinduced force microscopy. Phys. Rev. B 94, 195407 (2016).

33. Santos, S. et al. Energy dissipation distributions and dissipative atomic processes in amplitude modulation atomic force microscopy. Nanotechnology 23, 125401 (2012).

34. Anczykowski, B., Gotsmann, B., Fuchs, H., Cleveland, J. P. \& Elings, V. B. How to measure energy dissipation in dynamic mode atomic force microscopy. Appl. Surf. Sci. 140, 376-382 (1999).

35. Olmon, R. L. et al. Optical dielectric function of gold. Phys. Rev. B 86, 235147 (2012).

36. Zolotarev, V. M., Volchek, B. Z. \& Vlasova, E. N. Optical constants of industrial polymers in the IR region. Opt. Spectrosc. 101, 716-723 (2006).

37. Persson, B. N. J. Theory of the damping of excited molecules located above a metal surface. J. Phys. C Solid State Phys. 11, 4251-4269 (1978).
38. Persson, B. N. J. \& Lang, N. D. Electron-hole-pair quenching of excited states near a metal. Phys. Rev. B 26, 5409-5415 (1982).

39. de Melo e Souza, R., Kort-Kamp, W. J. M., Sigaud, C. \& Farina, C. Image method in the calculation of the van der Waals force between an atom and a conducting surface. Am. J. Phys. 81, 366-376 (2013).

40. Kim, B. \& Potma, E. O. Laser heating of cantilevered tips: implications for photoinduced force microscopy. Phys. Rev. B 100, 195416 (2019).

41. Persson, B. N. J. \& Persson, M. Damping of vibrations in molecules adsorbed on a metal surface. Surf. Sci. 97, 609-624 (1980).

\section{Acknowledgements}

The authors thank Thomas R. Albrecht and Sung Park for stimulating discussions and Derek Nowak for technical support on PiFM. The experimental and theoretical collaboration is supported by Chemistry at the Space-Time Limit (CaSTL) under Grant No. CHE-1414466. We thank University of Hail, Hail, Saudi Arabia for the Ph.D. Fellowship (to M.A.A.)

\section{Author contributions}

M.A.A. conducted experiments. M.A.A. and H.K.W. interpreted the results. S.M.A.U. built interferometer set-up and performed piezo calibration measurements. H.K.W. conceived and supervised the project. M.A.A. and H.K.W. wrote the manuscript.

\section{Competing interests}

H.K.W. is a co-founder of Molecular Vista Inc. M.A.A. and S.M.A.U. declare no competing interests.

\section{Additional information}

Supplementary information is available for this paper at https://doi.org/10.1038/s41467 020-19067-3.

Correspondence and requests for materials should be addressed to H.K.W.

Peer review information Nature Communications thanks the anonymous reviewer(s) for their contribution to the peer review of this work.

Reprints and permission information is available at http://www.nature.com/reprints

Publisher's note Springer Nature remains neutral with regard to jurisdictional claims in published maps and institutional affiliations.

pen Access This article is licensed under a Creative Commons Attribution 4.0 International License, which permits use, sharing, adaptation, distribution and reproduction in any medium or format, as long as you give appropriate credit to the original author(s) and the source, provide a link to the Creative Commons license, and indicate if changes were made. The images or other third party material in this article are included in the article's Creative Commons license, unless indicated otherwise in a credit line to the material. If material is not included in the article's Creative Commons license and your intended use is not permitted by statutory regulation or exceeds the permitted use, you will need to obtain permission directly from the copyright holder. To view a copy of this license, visit http://creativecommons.org/ licenses/by/4.0/.

(C) The Author(s) 2020 